\title{
Brillouin Scattering Based Distributed Fiber Optic Temperature Sensing for Fire Detection
}

\author{
Z. Liü, G. Ferrier*, X. Bao**, X. Zeng ${ }^{* *}$, Q. Yü ${ }^{* *}$ A. Kim* \\ *Fire Risk Management Program, National Research Council Canada, Ottawa, \\ Ontario, Canada, K1A 0R6, zhigang.liu@nrc.ca \\ ** Department of Physics, University of Ottawa, Ottawa, Ontario, Canada
}

\begin{abstract}
This paper presents a distributed fiber optic Brillouin scattering investigation, which involves measuring the Brillouin intensity versus time for real-time temperature measurements. This can be applied in practical fire detection schemes through the use of a threshold temperature that corresponds to the maximum Brillouin intensity. In the experiments, a $11 \mathrm{~km}$ fiber length was interrogated by $20 \mathrm{~ns}$ pulses ( $2 \mathrm{~m}$ spatial resolution) to monitor the temperature variations in a $10 \mathrm{~m}$ fiber region. The corresponding theory has confirmed the suitability of real-time intensity monitoring for temperature measurement.
\end{abstract}

KEY WORDS: fire detection, fiber optic temperature sensing, Brillouin scattering

\section{INTRODUCTION}

Optical fiber has been widely used for data transmission and physical sensing. In terms of physical sensing applications, optical fiber can be used for detecting variations in temperature, strain, and tensile force, as its refractive indices and geometric properties are correspondingly altered. Distributed fiber optic temperature sensing was first introduced for fire detection in the late 1980's [1,2]. Unlike the conventional fire detectors, the distributed optical fiber temperature sensor uses the entire optical fiber as the sensing medium. Temperature measurement can be made at any and every point along the fiber. The measured temperature is ranged from -160 to $600^{\circ} \mathrm{C}$, which is limited only by the durability of the fiber, or more specifically its primary coating. In comparison to conventional thermal detectors, the optical fiber sensor cable responds much more quickly to temperature due to its low mass. The fiber cable itself is strong, resilient, flexible and immune to all kinds of interference emissions. Conceivably, the location, size, and development of a fire can be determined with unmatched flexibility in measurement locations [2]. Distributed optical fiber temperature sensors based on Rayleigh and Raman scattering have been used to provide fire protection for those applications with difficult ambient conditions such as tunnels, underground railways and stations, conveyor lines, steelworks and petrochemical plants [2 5]. However, these distributed optical fiber temperature sensors have limited sensing ranges and their response times increase with the sensing length as well as the requirement for spatial and temperature resolutions due to weak light signals. The longest sensing range for the Rayleigh scattering system used for fire detection is up to $2 \mathrm{~km} \mathrm{[1]} \mathrm{and} \mathrm{for} \mathrm{the} \mathrm{Raman}$ scattering system is up to $4 \mathrm{~km}[6,7]$. The response time of the Raman system developed by Ericsson [6] is $4 \mathrm{~min}$ for a detected distance of $2 \mathrm{~km}$ with temperature resolution of $\pm 2^{\circ} \mathrm{C}$ and 
spatial resolution of $1 \mathrm{~m}$. It increases to $48 \mathrm{~min}$ if the detected distance is $4 \mathrm{~km}$ for the same temperature and spatial resolutions.

The distributed fiber optical temperature sensing system based on Brillouin scattering is seen as a potential replacement for the Raman scattering system for temperature measurement [8 10]. Physically, distributed fiber optic temperature sensing is based on the scattering of photons due to density fluctuations in the fiber. When the density fluctuations are dynamic, the scattered photons experience a frequency shift with a magnitude equal to the frequency of the density fluctuations. Through direct proportionality, the frequency shift is representative of the corresponding energy difference between the incident and scattered photons. This energy difference corresponds to the flux of acoustic phonons (sound waves) generated through electrostriction initiated by the strong input light waves entering the fiber, and the resulting scattering process is called Brillouin Scattering. Brillouin scattering based systems detect temperature changes by measuring the Brillouin backscattered intensity and its frequency shift as a function of temperature. Although Brillouin scattering yields signals that are approximately $10^{5}$ to $10^{6}$ times weaker than the incident light, they may be enhanced through a process known as Stimulated Brillouin Scattering (SBS). This can be achieved by sending light from two lasers into opposite ends of the sensing fiber. Through modulation of a pulsed laser, its amplification will occur at the expense of the second continuous wave (CW) laser, hereafter called the pump laser. This amplification process allows the fiber length to be significantly increased to approach $100 \mathrm{~km}$ due to improved signal-to-noise ratios resulting from nonlinear amplification in the fiber. The Brillouin system can also achieve much higher spatial and temperature resolutions for temperature measurement, compared to Rayleigh and Raman scattering systems.

Over the past decade, Brillouin scattering has become the most prominent scattering technique used for fiber optic sensing, where substantial improvements in fiber length and spatial resolution have been reported. In 1995, Bao et al. investigated fiber lengths as long as $51 \mathrm{~km}$ [11], while temperature and spatial resolutions of $\pm 1^{\circ} \mathrm{C}$ and $1 \mathrm{~m}$ respectively have been achieved for shorter fiber lengths of $1-3 \mathrm{~km}$ [9-13]. Although a number of scattering mechanisms have been proposed for distributed temperature sensing [6-8], the Brillouin scattering mechanism is thought to be most appropriate for fire detection due to its enormous potential for monitoring remote locations with increased temperature sensitivity. Fire protection specialists would benefit greatly from these advantages, so this paper presents Brillouin scattering on an experimental and theoretical level to demonstrate its suitability for fire detection. However, the data acquisition time of the Brillouin scattering system is directly proportional to the number of frequency steps within the scanning range, and Brillouin frequency has a linear dependence on temperature. Therefore, large temperature ranges require lengthy acquisition times. To illustrate this, consider a typical temperature range of $100^{\circ} \mathrm{C}$ corresponding to a $120 \mathrm{MHz}$ frequency range $\left(1.2 \mathrm{MHz} /{ }^{\circ} \mathrm{C}\right)$. At a laser wavelength of $1319 \mathrm{~nm}$, this process takes approximately 5-10 minutes. With increasing fiber lengths and shorter spatial resolutions, this processing time is further increased. Clearly, this scanning technique would be inappropriate for fire detection where much shorter response times are required.

By directly measuring the intensity of the Brillouin gain/loss signal versus time, the Brillouin system can respond more quickly to changes in fiber temperature, while maintaining good spatial and temperature resolutions and high signal-to-noise ratios (SNR $>20 \mathrm{~dB})$. In this 
paper, this temperature measurement technique is demonstrated through experimental and theoretical investigation and its potential for fire detection is discussed.

\section{THEORY}

When light propagates through an optical fiber, a small fraction is backscattered due to the Brillouin interaction between input photons and acoustic phonons, yielding either frequency down-shifted (Stokes) or up-shifted (anti-Stokes) photons. This corresponding frequency shift is known as the Brillouin frequency shift, $v_{B}$, and is given by:

$$
v_{B}=\frac{2 n V_{A}}{\lambda}
$$

where $\mathrm{n}$ is the refractive index of the fiber core, $V_{A}$ is the acoustic velocity, and $\lambda$ is the wavelength of the incident light. The Brillouin frequency shift is temperature dependent through variations in the refractive index of the fiber, and can be expressed as:

$$
v_{B}(T)=v_{B 0}+\frac{d v_{B}}{d T}\left(T-T_{0}\right)
$$

where $v_{B 0}$ is the Brillouin frequency at a reference temperature $T_{o}$. Current research has shown that the Brillouin frequency shift increases linearly with temperature, allowing equation (2) to be written as: [14, 15]

$$
v_{B}(T)=v_{B 0}+C_{T}\left(T-T_{0}\right)
$$

where $C_{T}$ is the frequency-temperature coefficient. The value of $C_{T}$ is determined by the fiber composition, laser wavelength, and additional fiber coatings and jackets [16]. Since equation (3) confirms the temperature dependence of the Brillouin frequency shift, the Brillouin scattering mechanism can be used as a basis for temperature measurement with Equation (3).

As previously mentioned, the intensity of a Brillouin scattered wave can be substantially increased through amplified Brillouin scattering, where a continuous wave $(\mathrm{CW})$ pump laser at frequency $v_{C W}$ interacts with a counter-propagating pulsed light wave at frequency $v_{p}$ within the fiber. The amount of amplification the pulsed beam experiences at the expense of the $\mathrm{CW}$ beam is defined as the Brillouin power gain, which is obtained from the solution to the coupled wave equations describing the pulsed and $\mathrm{CW}$ laser intensities. The equations are given by:

$$
\begin{aligned}
& \frac{d}{d z} I_{p}=-g\left(v_{C W}, v_{p}\right) I_{C W} I_{p}-\alpha I_{p} \\
& \frac{d}{d z} I_{C W}=-g\left(v_{C W}, v_{p}\right) I_{C W} I_{p}+\alpha I_{C W}
\end{aligned}
$$


where $I_{C W}$ and $I_{p}$ are the pump and pulsed laser intensities, $\alpha$ is the fiber attenuation coefficient, and $g\left(v_{C W}, v_{p}\right)$ is the Brillouin gain factor, which is dependent on both the laser input frequencies and the Brillouin frequency shift of the fiber. The solution for the Brillouin power gain has been determined through perturbation methods by Bao et al.[9], while properly accounting for large input powers and long fiber lengths, and is provided here in terms of the pump laser intensity $I_{C W}(z)$ as:

$$
\frac{I_{C W}(z)}{I_{C W}(z+u)}=\exp \left[\frac{g\left(v_{C W}, v_{p}\right) I_{p}(0) e^{\beta}}{\alpha}\left(\frac{E_{2}\left(\beta e^{\alpha z}\right)}{e^{\alpha z}}-\frac{E_{2}\left(\beta e^{\alpha(z+u)}\right)}{e^{\alpha(z+u)}}\right)-\alpha u\right]
$$

where $u$ represents the spatial resolution, $I_{p}(0)$ is the input pulse power, $\mathrm{E}_{2}\left(\mathrm{z}_{0}\right)$ [17] is the second order exponential integral defined as $E_{2}\left(z_{0}\right)=\int_{1}^{\infty} \frac{\exp \left(-z_{0} t\right)}{t^{2}} d t$, $\beta=\frac{g\left(v_{C W}, v_{p}\right) I_{C W}(L) e^{-\alpha L}}{\alpha}$, and $L$ is the fiber length.

The main limitation of the Brillouin power gain solution arises from the spatial resolution requirement. As this solution was obtained strictly at the steady state condition, it will provide a reasonable approximation only when the pulse width remains longer than the phonon lifetime of $10 \mathrm{~ns}$ (1 m spatial resolution). Abiding by this large spatial resolution requirement, Bao properly assumed a Lorentzian Brillouin gain profile in determining the solution above. For most fire applications this restriction will not be problematic, especially when monitoring remote locations $(>50 \mathrm{~km})$ where temperature resolution is of greater concern. However, it may be desirable in some applications to have spatial resolutions near $1 \mathrm{~m}$, where a Lorentzian distribution would not completely describe the optical process because large linewidth increases near the phonon lifetime significantly changes the spectral shape. Consequently, the pseudo-Voigt profile has been proposed to handle such situations, and represents a combination between the Lorentzian and Gaussian profiles and is written as: [13]

$$
g\left(v_{C W}, v_{p}\right)=\gamma g_{B M}\left(\frac{\delta}{1+\frac{4\left(v_{C W}-v_{p}-v_{B}\right)^{2}}{\Delta v^{2}}}+(1-\delta) e^{-4 \ln 2 \frac{\left(v_{C W}-v_{p}-v_{B}\right)^{2}}{\Delta v^{2}}}\right)
$$

where $\gamma$ is the polarization factor, $v_{B}$ is the Brillouin frequency, $v_{C W}$ and $v_{p}$ are the pump and pulsed laser frequencies respectively, $\Delta v$ is the linewidth of the Brillouin gain profile, $\delta$ is the pseudo-Voigt shape factor (Fully Lorentzian: $\delta=1$; Fully Gaussian: $\delta=0$ ), 
and $g_{B M}$ is the maximum gain coefficient given by:

$$
g_{B M}=\frac{2 \pi n^{7} P_{12}{ }^{2}}{c \lambda^{2} \rho V_{a} \Delta v}
$$

where $P_{12}$ is the longitudinal elasto-optic coefficient, $\rho$ is the material density and $c$ is the light speed.

As indicated in Equations (6) and (7), the Brillouin power gain has a frequency dependence via the Brillouin gain factor, $g\left(v_{C W}, v_{p}\right)$. In addition, there is a clear exponential relationship between the Brillouin power gain and the Brillouin gain spectrum. Therefore, using equation (7) as the general form of $g\left(v_{C W}, v_{p}\right)$, it can finally be shown that the Brillouin power gain is maximized when the Brillouin frequency shift at a unique fiber location is equal to the frequency difference between the $\mathrm{CW}$ pump and pulsed waves ( $v_{B}=$ $v_{C W}-v_{p}$ ) for that local temperature condition. The maximum Brillouin power gain is determined by recording the pulsed laser power over time, and can be used to trigger an alarm signal for fire detection purposes. This can be achieved by setting up a threshold temperature, $T_{t h}$, that corresponds to the maximum Brillouin power gain, based on specified fire detection conditions. Experimentally, the frequency difference between the pump and pulsed lasers may be tuned to match the Brillouin frequency of the fiber at a known threshold temperature, $T_{t h}$, obtained by equation (3). When the fiber temperature increases, the Brillouin power gain will increase and become a maximum when the temperature equals $T_{t h}$. and at this temperature, an alarm signal would subsequently be triggered indicating that this threshold temperature has been reached. The temperature tolerance of an optical fiber is mainly limited to its surrounding fiber jacket, which in many circumstances can withstand temperatures exceeding $100^{\circ} \mathrm{C}$. For this reason, high threshold temperatures may conceivably be employed for increased reliability, as $T_{t h}$ would then be distinctively greater than temperatures of most ambient environments.

The response time of the Brillouin system to changes in fiber temperature is dependent on the pulse propagation time (Equation (9)), data processing time, and the response time of the fiber core, cladding, and jacket. The pulse propagation time is very short due to the extremely high velocity of photons in optical fiber $\left(\mathrm{c} / \mathrm{n}=2 \times 10^{8} \mathrm{~m} / \mathrm{s}\right)$, and is determined simply by:

$$
t=\frac{2 n L}{c}
$$

For a $10 \mathrm{~km}$ fiber length $(L)$, the time between consecutive pulses is approximately $100 \mu \mathrm{s}$.

The data processing time is collectively determined from the electronic response times, and the number of time-domain averages per waveform. The electronic response time is a few milliseconds, while more time-domain averages require longer data processing times. It is 
vitally important to minimize the time-domain averages for fastest response times. However, in practice, some averaging is required in order to reduce optical and electrical noise. Therefore, the number of averages should be optimally compromised for best performance. The response time of silica is practically negligible, as the response of bound electrons to optical fields requires only a few nanoseconds. However, the time required to completely heat the sensing fiber to a constant temperature is minimized only when the sensing fiber is short, or looped, and isolated from the adjacent fiber.

The delay time between the launch of the pulsed beam and those regions where Brillouin scattering is amplified will provide the positional information for fire detection. As the pulse propagates along the fiber, the Brillouin frequency shift associated with different fiber regions may be determined. In addition, the use of a pulsed laser beam allows the Brillouin system to achieve a short spatial resolution. This is because amplification can only occur within the region of coincidence of the two light waves at any one time, when the Brillouin frequency is matched. Equation (9) confirms that temporally short pulses are also physically short. This suggests that the pulsed beam amplification only occurs within a small fiber region at any given instant in time. Ultimately, the pulse width determines the temporal and hence spatial resolution of the Brillouin system.

\section{EXPERIMENTAL APPARATUS AND PROCEDURE}

The configuration of the Brillouin sensor system is shown in Figure 1. It consists of digital, electronic, and optical components working collaboratively to obtain the Brillouin signal. The optical dynamics are manipulated by means of power supplies, amplifiers, and attenuators while being interpreted and delivered to the computer via digital connections. In addition, three photodetectors are used to convert the optical signals into electronic signals. As previously mentioned, the Brillouin amplification is created through the interaction of two counter-propagating laser beams within the sensing fiber. To collect the signal properly, the system must perform a number of tasks simultaneously. These include frequency stabilization, polarization control, and both the collection and processing of data. This is partially achieved with an optical circulator and couplers, which route portions of the optical signal for task management. The high ratio couplers ensure that high powers are available for Brillouin interaction within the fiber, while the low powered signals are used for frequency stabilization, power monitoring, and oscilloscope display, which can be replaced by a computer. A frequency counter was used to continuously monitor the frequency difference between the two $\mathrm{CW} \mathrm{Nd:YAG} \mathrm{ring} \mathrm{lasers,} \mathrm{which} \mathrm{operated} \mathrm{at} \mathrm{a} \mathrm{wavelength} \mathrm{near}$ $1319 \mathrm{~nm}$. 


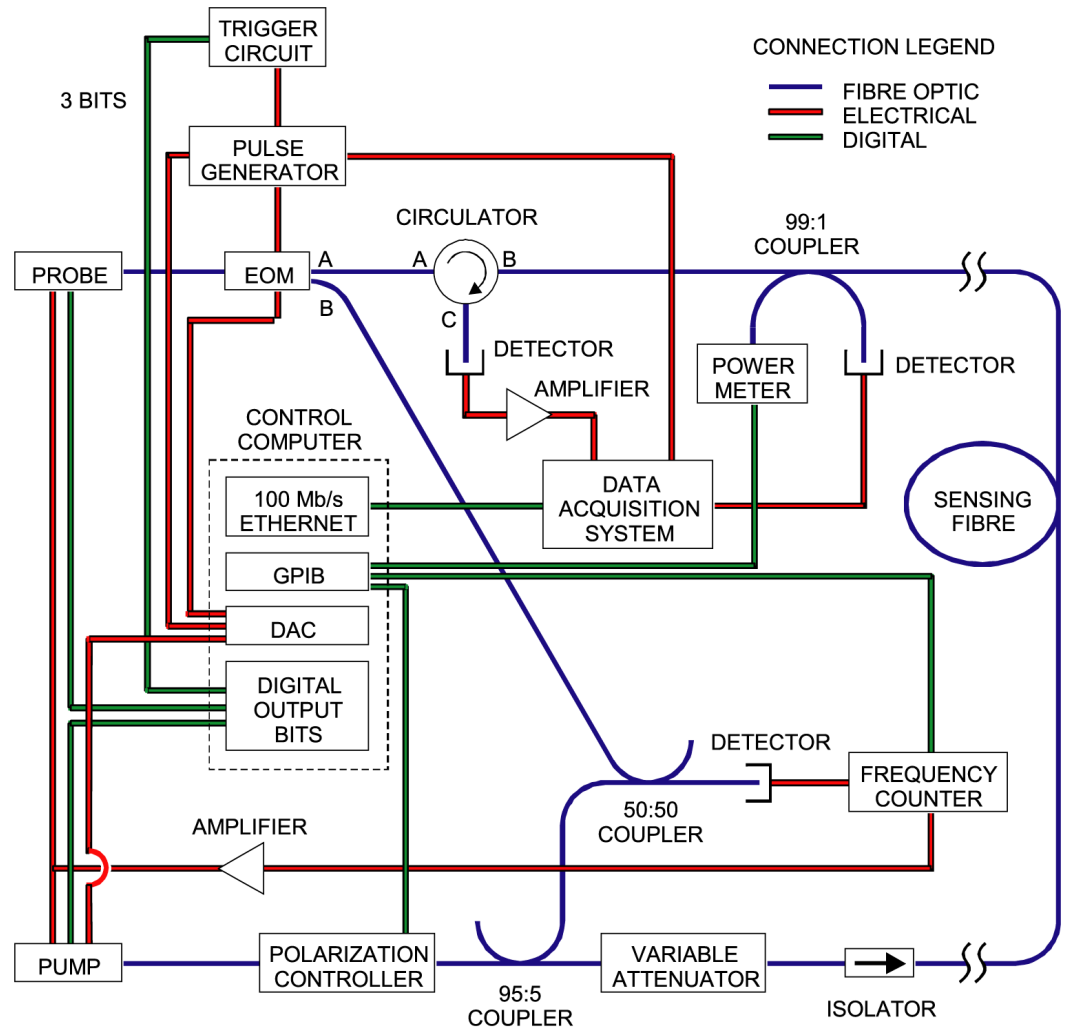

Figure 1: $\quad$ Configuration of the distributed Brillouin system

The progress of the optical signal begins with the pulsed laser, when its beam is initially pulsed with amplitude modulation using an electro-optic modulator (EOM). The pulsed beam then enters the circulator, which sends most of the signal toward the sensing fiber. The short pulsed beam ranges in time $20 \mathrm{~ns}$ that corresponds to $2 \mathrm{~m}$ spatial resolution. At the same time, the light from a counter-propagating CW pump laser is directed through a polarization controller that optimizes its polarization state, and is then attenuated to a maximum power of approximately $10 \mathrm{~mW}$, where it then passes through an optical isolator and enters the sensing fiber for Brillouin interaction with the pulsed beam. The optical isolator serves to prevent the pulsed beam from entering the pump laser where laser destabilization would otherwise happen. After the Brillouin interaction, the newly created Brillouin loss signal continues its progression through the 99:1 coupler for power monitoring, enters the circulator, and finally exits into the digitizer, where the data is collected and analyzed.

The following experiment involves monitoring the temperature conditions of an $11 \mathrm{~km}$ fiber purely by monitoring the pump beam intensity versus time. The fiber used in experiments was single SMF-28 communication fiber. A looped $10 \mathrm{~m}$ fiber section was subjected to 
temperature variations, while the remaining fiber was left at room temperature. This was done to demonstrate the potential of the system at a $2 \mathrm{~m}$ spatial resolution. The temperature varying fiber, hereafter called the "hot spot", was positioned approximately $8 \mathrm{~km}$ from the CW pump laser. The experiment begins by having the frequency difference between the lasers locked to a value of $12860 \mathrm{MHz}$, which corresponds to a preset threshold temperature. A heat gun was used to increase the temperature of the "hot spot", and was placed within close proximity for increased heat intensity. A thermocouple was then placed near the "hot spot", while both were thermally insulated to minimize the heat loss. Finally, the Brillouin signals were observed and recorded using a digitizing oscilloscope at a rate of approximately 2 seconds per waveform. By limiting the number of time domain averages to just 10 , this data acquisition rate was accessible.

Brillouin Shift versus Temperature

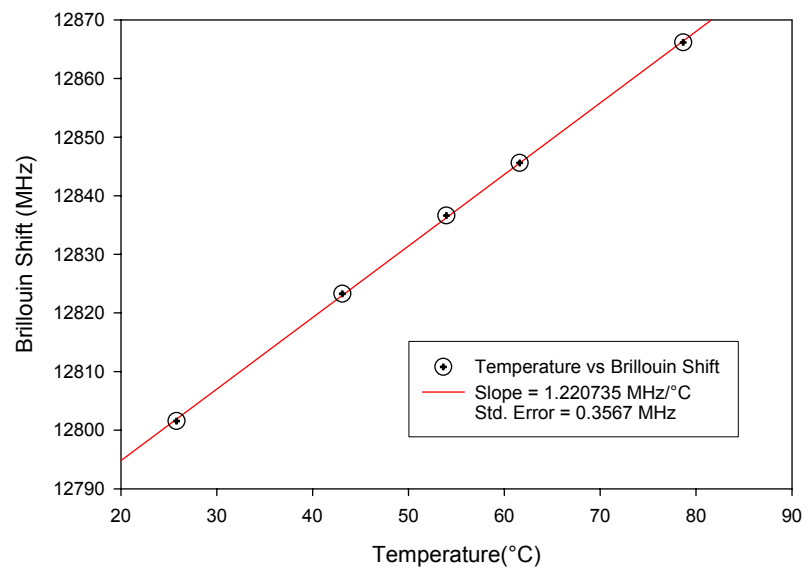

Figure 2: A temperature-frequency calibration confirms a linear relationship between the two variables. The slope provides an accurate value for $\mathrm{C}_{\mathrm{T}}$, over the operating temperature range.

\section{RESULTS AND DISCUSSION}

The Brillouin intensities of the $10 \mathrm{~m}$ "hot spot" have been acquired for several temperatures in the $40-80^{\circ} \mathrm{C}$ temperature range over a 20 second timeframe. The threshold temperature corresponding to a $12860 \mathrm{MHz}$ Brillouin frequency has been determined experimentally from the Brillouin intensity results, and theoretically using equation (3). The reference data, $v_{B 0}$ and $T_{0}$, are obtained from a temperature-frequency calibration, and from Figure 2 , the temperature-frequency relationship can be formulated as:

$$
v_{B}(T)=12796+1.22(T-20)
$$

From this equation, a $72.4^{\circ} \mathrm{C}$ temperature corresponds to a $12860 \mathrm{MHz}$ Brillouin frequency. For $C_{T}$, the value of $1.22 \mathrm{MHz} /{ }^{\circ} \mathrm{C}$ is regarded as standard for SMF-28 single mode fiber $[13,16]$. Figure 3 shows the variation of the corresponding Brillouin intensity as the function of the fiber temperature with the heating time. 9 fiber temperatures were recorded 
during heating period and each Brillouin intensity curve in Figure 3 corresponded to one fiber temperature. The Brillouin scattering intensity quickly increased as the fiber temperature increased. The response time was in second range.

\section{"Hot Spot" Brillouin Gain Variation $\left(v_{B}=12860 \mathrm{MHz}\right.$, sensing fiber $=10 \mathrm{~m}$, total fiber length $\left.=11 \mathrm{~km}\right)$}

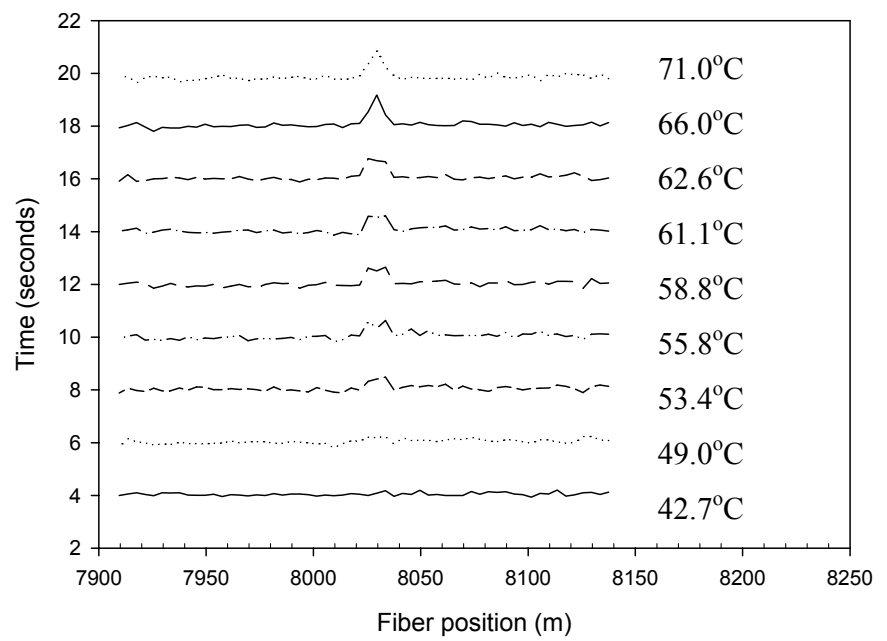

Figure 3: Variation of the Brillouin power gain with time. The fiber position is the distance from the CW pump laser. The "hot spot" Brillouin gain increases during heating, and becomes a maximum after $18 \mathrm{~s}$. The width of the "hot spot" is $10 \mathrm{~m}$.

Figure 4 shows the corresponding change in normalized Brillouin intensity gain with temperature. The maximum Brillouin gain was obtained at a temperature of $66^{\circ} \mathrm{C}$. Consequently, a $6-7^{\circ} \mathrm{C}$ temperature uncertainty is apparent from our experiment. This is mainly attributed to a relatively long $2 \mathrm{~m}$ spatial resolution compared with the $10 \mathrm{~m}$ "hot spot", combined with the slowly changing temperature gradients existing near its boundaries, resulting in non-uniform temperature changes throughout the entire "hot spot" region. In Figure 3, this phenomenon is observed as the central region buckles relative to the "hot spot" boundaries. Since the Brillouin detection system averages the temperature over a given spatial resolution to obtain temperature information, the Brillouin gain throughout the $10 \mathrm{~m}$ has been somewhat diminished. In addition, by using only 10 time-domain averages, the noise levels increase and hence influences the Brillouin gain data, especially when the gain is low. This would explain the non-uniform temperature variations shown in Figure 4, where the Brillouin gain changes are small. To practically limit the temperature uncertainty, it would seem that more time-domain averages are required to filter out excess optical and electrical noise. However, in our previous temperature threshold experiment [18], only 8 time-domain averages were required to monitor transient temperature measurements in a 22 $\mathrm{km}$ single mode fiber, where a $100 \mathrm{~m}$ sensing length was monitored using a $50 \mathrm{~ns}$ pulse $(5 \mathrm{~m}$ spatial resolution). The larger 100:5 length ratio creates a more uniform temperature distribution throughout the majority of the sensing fiber length. In this way, the pulse energy is used in a more constructive manner in maintaining temperature integrity. Consequently, this length ratio has proven to be an important parameter in real-time temperature monitoring. 
As a result, the amplification of the Brillouin scattering intensity in our previous experiments reached its maximum when the fiber was heated to its threshold temperature. Any further increase in fiber temperature resulted in the decrease in the Brillouin power gain.

Normalized Brillouin Gain versus Temperature $\left(v_{B}=12860 \mathrm{MHz}\right)$

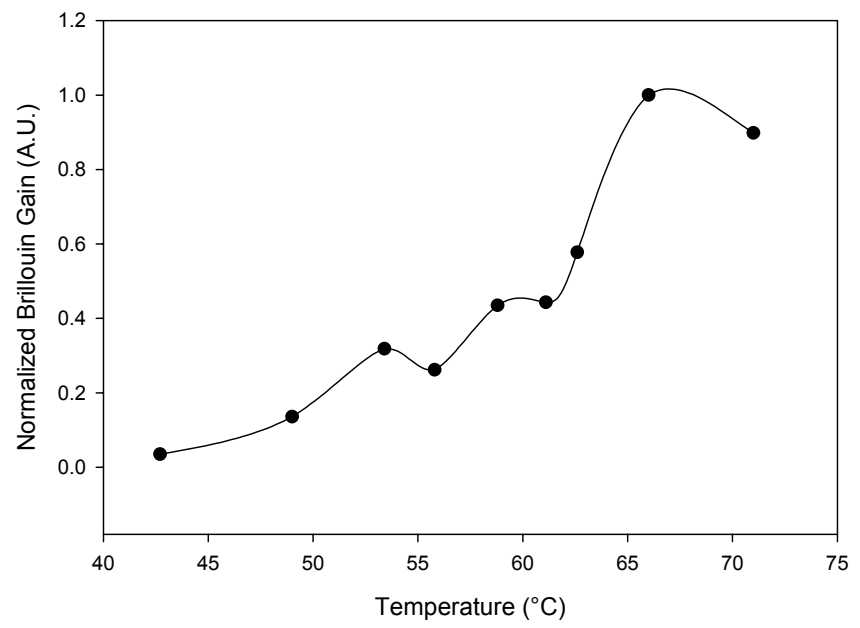

Figure 4:Variation of normalized Brillouin power gain with "hot spot" temperature. During heating, the "hot spot" Brillouin gain increases until the threshold temperature $\left(\mathrm{T}_{t h}\right)$ of $66^{\circ} \mathrm{C}$ is reached. Continued heating $\left(\mathrm{T}>66^{\circ} \mathrm{C}\right)$ results in diminished gains as the Brillouin frequency corresponding to $\mathrm{T}_{t h}$ is no longer matched.

With this idea in mind, the progress of this research should continue in the direction of improving spatial resolutions, and to establish operating system conditions corresponding to the optimum signal-to-noise ratios (SNR) achieved for different fiber lengths. Although the data acquisition time was fast, much of the preliminary time was spent finding optimum power levels by manually adjusting the attenuator and power supply settings. The inclusion of flow charts for this purpose will help to alleviate this problem. Currently, the main limitation of the distributed system lies in the balance between short spatial resolution and long sensing lengths, and in some fire detection applications, it would be desirable to use complex fiber geometries where long sensing lengths may be ineffective. Therefore, spatial resolution improvements would allow shorter sensing lengths to be applied, while maintaining large length ratios and improved temperature resolution. This does not pose immediate problems, as $1-5^{\circ} \mathrm{C}$ temperature resolutions are adequately sensitive for most fire sensing applications. In addition, the fiber reaction time can be substantially improved by changing the threshold temperature itself. For instance, a threshold temperature of $70^{\circ} \mathrm{C}$ is substantially above most ambient temperatures, while its detection from $40^{\circ} \mathrm{C}$ can be done in approximately 20 seconds, as demonstrated in this experiment. However, smaller differences between the threshold and ambient temperatures would allow the alarm to be triggered even faster at the expense of marginal decreases in temperature sensitivity. As the demand for increased fiber length and shorter spatial resolution grows, further investigations will be conducted to realize the true potential of distributed Brillouin sensing for fire sensing applications. 


\section{CONCLUSION}

This paper has aimed to demonstrate the competence of the distributed Brillouin system in rapidly detecting temperature changes for fire detection applications. In practice, reliable fire detection requires remote surveillance combined with short response times to minimize and isolate the impact of a potentially hazardous fire. Distributed fiber optic Brillouin sensing systems appear to be most ideally suited for this application, and with further investigations may have the potential to eventually replace the current Raman and Rayleigh scattering mechanisms as the most dominant, and accurate, fire sensing technique. Including the various environments mentioned in this report, Brillouin scattering systems also have the potential to be used to monitor valuable telecommunication facilities and nuclear plants.

The experiments reported in this paper have successfully incorporated a long fiber length (11 $\mathrm{km})$ for monitoring the temperature variations of a relatively small sensing length $(10 \mathrm{~m})$. The data acquisition time has been substantially improved by monitoring the Brillouin gain intensity directly, rather than sweeping through a range of frequencies, thus allowing measurements to be made in real-time. The maximum Brillouin power gain that corresponds to a threshold temperature, $T_{t h}$, can be used to trigger an alarm signal for fire detection purposes. The corresponding theory has confirmed the suitability of real-time intensity monitoring for temperature measurement.

\section{REFERENCES}

1. Meacham, B. J., "International Developments in Fire Sensor Technology," J. of Fire Protection Engineering, 6 (2), 89-98, 1994.

2. Morgan, A., "New Fire Detection Concepts with Fibre Optics Technology," Fire Safety Engineering, 35-37, 2000.

3. Iida, O., Onoda, D., Kono, S., Hshiba, K. and Ohsawa, S., "Expansion of Measuring Range for a Fiber-Optic Distributed Temperature Sensor and Applications to Commercial Plants," IMTC'94, May 1994, Hamamatsu, Japan.

4. Wang, A., Liu, W., Li, X., Yue, C., Wang, Y., Wang, Q., and Cai, X., "Distributed Optical Fiber Temperature Detecting and Alarm System," $12^{\text {th }}$ International Conference on Automatic Fire Detection, March 2001, Gaithersburg, USA.

5. Maegerle, R., "Fire Protection Systems for Traffic Tunnels Under Test," $12^{\text {th }}$ International Conference on Automatic Fire Detection, March 2001, Gaithersburg, USA.

6. Ericsson Network Engineering AB, Sundbyberg, Sweden, "Distributed Temperature Measurement Using Optical Fibres," - System Document, 1994.

7. Basnett, T. and Barber, S. J., "Distributed Temperature Measurement with Optical Fibres," - System document, Cossor Electronics Ltd, Harlow, Essex, 1994.

8. Culverhouse, D., Farahi, F., Pannell, C.N., and Jackson, D.A., "Potential of Stimulated Brillouin Scattering as Sensing Mechanism for Distributed Temperature Sensors," Electronics Letters, Vol. 25, No. 14, 1989.

9. Bao, X., Webb, D. J., and Jackson, D. A., "32-km Distributed Temperature Sensor Based on Brillouin Loss in an Optical Fiber," Optics Letter, Vol. 18, No.18, September 1993, pp.1561-1563

10. Thevenaz, L., Nikles, M., Fellay, A., Facchini, M. and Robert, P., "Truly 
Distributed Strain and Temperature Sensing Using Embedded Optical Fibers," SPIE proceedings, 3330:301-314, 1998.

11. Bao, X., Dhilwayo, J., Heron, N., Webb, D. J., and Jackson, D. A., "Experimental and Theoretical Studies on a Distributed Temperature Sensor Based on Brillouin Scattering," J. of Lightwave Technology, Vol. 13, No. 7, 1995.

12. Fellay, A., Thevenaz, L., Facchini, M., Nickles, M., and Robert, P., "Distributed Sensing Using Stimulated Brillouin Scattering towards Ultimate Resolution," Optical Fiber Sensors, Vol. 16, 324-327, 1997.

13. Smith, J., "Characterization of the Brillouin Loss Spectrum for Simultaneous Distributed Sensing of Strain and Temperature," M. Sc. Thesis, University of New Brunswick, 1999.

14. Bao, X., Brown, A., DeMerchant, M. and Smith, J., "Characterization of the Brillouin-Loss Spectrum of Single-Mode Fibers by Use of very Short $(<10$-ns) Pulses," Optics Letters, Vol. 24, No.8, 510-512, 1999.

15. Garus, D., Gogalla, T., Krebber, K., and Schliep, F., "Brillouin Optical-fiber Frequency Domain Analysis for Distributed Temperature and Strain Measurements," J. of Lightwave Technology, Vol. 15, No. 4, 1997.

16. DeMerchant, M., "Distributed Strain Sensing for Civil Engineering Applications," Ph.D. Thesis, University of New Brunswick, 2001.

17. Abramowitz, M., and Stegun I., Handbook of Mathematical Functions, Dover, 228269, 1965.

18. Bao, X., Webb, D. J., and Jackson, D. A., "Distributed Temperature Sensor Based on Brillouin Loss in an Optical Fiber for Transient Threshold Monitoring," Canadian Journal of Physics, Vol. 74, 1-3, 1996. 